1. Offer G, Moos C, Starr R. A new protein of the thick filaments of vertebrate skeletal myofibrils: extraction, purification and characterization. Mol Biol 1973 ; 74 : 653-76.

2. Einheber S, Fischman DA. Isolation and characterization of a cDNA clone encoding avian skeletal muscle C-protein: an intracellular member of the immunoglobulin superfamily Proc Nat Acad Sci USA 1990; $87: 2157-61$.

3. Yamamoto K, Moos C. The C-proteins of rabbi red, white, and cardiac muscles. J Biol Chem 1983 $258: 8395-401$.

4. Gautel M, Zuffardi O, Freiburg A, Labeit S Phosphorylation switches specific for the cardia isoform of myosin binding protein $\mathrm{C}$ : a modula tor of cardiac contraction? EMBO J $1995 ; 14$ : 1952-60.

5. Moos C, Feng INM. Effect of C-Protein on actomyosin ATPase. Biochim Biophys Acta $1980 ; 632$ 141-9.

6. Hofmann PA, Hartzell HC, Moss RL. Alterations in $\mathrm{Ca}^{2+}$ sensitive tension due to partial extraction of C-protein from rat skinned cardiac myocyes and rabbit skeletal muscle fibers. $J$ Gen Physiol 1991 ; 97 : 1141-63.

7. Hofmann PA, Greaser ML, Moos RL. C-protein limits shortening velocity of rabbit skeletal muscle fibres at low levels of $\mathrm{Ca}^{2+}$ activation. J Physiol $1991 ; 439: 701-15$

8. Schlender KK, Bean LJ. Phosphorylation of chicken cardiac C-protein by calcium/calmodulin-dependent protein kinase II. J Biol Chem 1991 266 : 2811-7

9. Carrier L, Guicheney P, Schwartz K. La cardiomyopathie hypertrophique familiale : maladie du sarcomère cardiaque ? médecine/sciences 1995 $11: 1685-93$.

10. Bonne G, Carrier L, Bercovici J, Cruaud C, Richard P, Hainque B, Gautel M, Labeit S, James M

Weissenbach J, Vosberg HP, Fiszman M, Komajda M, Schwartz K. A splice acceptor site mutation in the cardiac myosin binding protein-C gene is associated with familial hypertrophic cardiomyopathy. Nature Genet 1996 ; Il (sous presse).

11. Dreyfus JC, Akli S, Poenaru L Maladies de Tay-Sachs et de Sandhoff. Les déficits en $\beta$-hexosaminidases, modèles des maladies des lysosomes. médecine/sciences $1992 ; 8: 797-803$.

\title{
Les mutants déficients en rapsyne révèlent un rôle essentiel inattendu de cette protéine
}

La jonction neuromusculaire, qui ne couvre que $1 / 10000$ de la totalité de la surface musculaire, est un paradigme de spécialisation membranaire. Tous les composants moléculaires nécessaires à la transmission synaptique, en particulier le récepteur nicotinique de l'acétylcholine (AchR), sont concentrés au niveau de ce site. Le puzzle des différents acteurs impliqués dans ce rassemblement spécifique, comme l' $\alpha$-dystroglycane, membre du complexe de protéines associées à la dystrophine (DGC) et elle-même récepteur de l'agrine $\left(\mathrm{m} / \mathrm{s} n^{\circ} 10\right.$, vol. 10, p. 1042), se met en place progressivement sans que l'on puisse désigner celui d'entre eux qui joue le rôle fondateur. Il semble que la rapsyne (receptor-associated protein at the synapse), une protéine de $43 \mathrm{kDa}$, soit désormais le candidat favori. Des expériences de reconstitution moléculaire par transfection de différents vecteurs d'expression dans des modèles de culture cellulaire avaient déjà suggéré ce rôle. On peut, par exemple, mentionner le récent travail d'Apel et al. [1] qui montre que la rapsyne, synthétisée sous le contrôle d'un vecteur d'expression transfecté dans des cellules fibroblastiques de caille dépourvues de tout l'arsenal protéique synaptique, est à elle seule capable de se distribuer en agrégats. Si un vecteur d'expression de la dystroglycane ou de l'AchR est ajouté à celui de la rapsyne, la dystroglycane et l'AchR synthétisés se regroupent également dans ces mêmes agrégats alors que, si l'on ne transfecte pas le gène codant pour la rapsyne, ces deux protéines adoptent une répartition homogène. En outre, dystroglycane et AchR ne se regroupent pas isolément (Tableau I). La rapsyne semble donc essentielle pour l'agrégation des récepteurs de l'acétylcholine et peut être le relais de l'interaction entre les AchR et le complexe DGC. De plus, l'interaction entre la rapsyne et la dystroglycane semble indépendante des AchR.

Une collaboration entre les laboratoires de Merlie et Sanes (USA) a permis d'obtenir, quant au rôle précis in vivo de la rapsyne, des informations supplémentaires essentielles

\begin{tabular}{|lc|}
\hline \multicolumn{2}{|c|}{ Tableau I } \\
\hline Vecteurs d'expression transfectés & Organisation en agrégats \\
\hline Rapsyne & + \\
Dystroglycane & - \\
AchR & - \\
Rapsyne + dystroglycane & + \\
Rapsyne + AchR & + \\
Dystroglycane + AchR & - \\
\hline
\end{tabular}


[2]. Les souris mutantes, obtenues par recombinaison homologue, ne produisant plus la rapsyne meurent peu de temps après la naissance. L'analyse des jonctions neuromusculaires de ces animaux révèle l'absence totale d'agrégation des AchR. S'il ne s'agissait là que des seules anomalies des souris déficientes en rapsyne, il n'y aurait que confirmation du rôle essentiel joué par la rapsyne dans l'agrégation des AchR. Mais la fonction de la rapsyne semble ne pas s'arrêter là. En effet, les synapses musculaires des souris mutantes sont dépourvues d'utrophine et les $\alpha$ - et $\beta$-dystroglycanes ainsi que l' $\alpha$-syntrophine, trois protéines normalement concentrées à la synapse, sont, chez les souris rapsyne -/-, réparties de façon équivalente dans les aires synaptiques et extrasynaptiques, suggérant un rôle essentiel de la rapsyne dans l'organisation du complexe cytosquelettique post-synaptique. Toutefois, ces observations ne suffisent pas à comprendre si une liaison directe s'opère entre rapsyne et membres du complexe DGC. Des anomalies présynaptiques sont également observées chez les mutants : si les souris normales et mutantes présentent une apposition normale des membranes pré- et post-synaptiques, les terminaisons nerveuses des souris mutantes sont anormalement longues et dépourvues des arborisations caractéristiques des terminaisons normales. Cette anomalie suggère que l'organisation du complexe post-synaptique de la plaque motrice, perturbée par le déficit en rapsyne, joue un rôle inducteur dans la morphogenèse des terminaisons nerveuses présynaptiques.

H.G.

1. Apel ED, Roberds SL, Campbell KP, Merlie JP. Rapsyn may function as a link between the acetylcholine and the agrin-binding dystrophin-associated glycoprotein complex. Neuron $1995 ; 15$ : 11526.

2. Gautam M, Noakes PG, Mudd J, Nichol M, Chu GC, Sanes JR, Merlie JP. Failure of postsynaptic specialization to develop at neuromuscular junctions of rapsyn-deficient mice. Nature 1995 ; 377 : 232-6. 